\title{
Pins and Posters: Paradigms for Content Publication on Situated Displays
}

\author{
Rui José, Hélder Pinto, Bruno Silva and Ana Melro \\ Centro Algoritmi, University of Minho, Portugal \\ Email: \{rui, helder, bruno.silva, amelro\}@dsi.uminho.pt
}

\begin{abstract}
Public display systems are still far from being a communication medium that people can appropriate to serve diverse communication goals. Moving towards open displays will require new publication paradigms that can overcome the challenges of meaningful engagement and enable users to fully understand and control the entire publication process. In this paper, we report on the study of two novel and complimentary communication paradigms for public displays inspired by the metaphors of pin badges and posters. The study is grounded on a 6 month deployment of Instant Places across 10 displays in diverse urban locations. We have collected user and system data regarding the emerging practices around these publication paradigms. The findings from this study constitute a novel contribution towards understanding the elements that may drive user-generated content in networks of urban displays, informing the design of new tools and procedures for situated publication in public displays.
\end{abstract}

Keywords-Public displays; User-generated content; Situatedness

\section{INTRODUCTION}

Digital public displays of various sizes and form factors constitute an increasingly important element of our technological landscape with an immense potential to radically change communication in public and semipublic spaces. We envision this trend to progressively enable us to move away from a world of closed display networks that are operated as multiple isolated islands to scenarios in which large-scale networks of pervasive public displays and associated sensors are open to applications and content from many sources [1]. In these scenarios, displays would become a communication medium ready to be appropriated by users for whatever communication goals they could find valuable. Usergenerated content would become a commodity that could drive entirely new services and business models around public displays.

However, existing display systems are still far from reaching their potential as an open communication medium. Observational studies have shown that people tend to perceive their content as not relevant and learn to ignore the displays [2]. Enticing people to participate and generate regular contributions to public displays has itself revealed to be a recurrent problem. Huang and Mynatt [3] observed that individuals tend not to be motivated to supply content, or else have difficulty identifying appropriate content. Müller et al. [4] describe how public displays may be perceived as a stage in which people will only act if they feel confident about their actions and in full control over the presentation of self. Memarovic et al. show how public displays must be seen as part of a communicative ecology in which they are mainly associated with content that addresses community and its interests [5].

These studies seem to suggest a strong gap between the potential of public displays as a communication medium and the necessary grasp of the respective communication process. While this also requires mastering interaction techniques, it clearly goes beyond the mere issue of how to interact. Consider the simple example of sending an SMS. When being sent from one person to the other the context is well defined by the service itself and by the shared context between those people. On the contrary, when sending an SMS to a public display, and even though interaction remains the same, we lose any references upon which to base our expectations on how that content is going to be seen, interpreted or repurposed. A similar reasoning applies to the use of social networks, such as Facebook or Twitter, as publication tools. While it may be simple to enable content from these social networks to flow to public displays, it clearly remains an open issue how to extend the publication paradigms from those systems into the specificities of content sharing on public displays.

We thus envision that overcoming the challenges of meaningful communication using public displays will require specific publication paradigms that enable people to control and reason about the way content is published.

\section{A. Objectives and approach}

In this research, we study two novel content publication paradigms for public displays inspired by existing forms of personal self-expression: posters and pin badges. The study is based on our own development of Instant Places, a novel screen media system that enables registered users to publish content to the displays using either posters or pin badges. The paper reports on a specific Instant Places deployment that occurred over a period of 6 months and involved 10 displays across 7 distinct locations. Place owners across those locations were also able to create content, and accept or decline content published by others to their displays. We have conducted surveys and interviews with users and place owners, as well as an analysis of system data. Our findings address the general publication practices promoted by each of the publication paradigms, the situatedness strategies for linking content 
with appropriate presentation contexts and the social negotiations around moderation and trust. This constitutes a novel contribution towards understanding the tools and practices that may drive the emergence of user-generated content in networks of urban displays.

\section{II.RELATED WORK}

The challenge of situated engagement with public displays has been previously approached from many diverse perspectives. Schroeter et al. [6] have shown how crucial it is to find the right combination between people, content and location in order to achieve the right type of situated engagement with public displays. This view is in line with our own motivation. Our goal is precisely to explore publication paradigms that constitute particular ways in which to frame valuable connections between those same elements.

Publication practices around non-digital displays have already been studied as a design inspiration for the emergence of new practices around digital displays. Alt et al. [7] have explored traditional public notice areas to uncover some of the practices behind their operation and discuss their role in the design of future generations of globally networked public displays.

Publication practices around large scale networks of public displays have been studied by Friday et al. [8] in a long-term analysis of the e-campus deployment at Lancaster University. This work has focused on the display managers' perspective and highlighted the diversity of requirements that different stakeholders, even in a single organization, may have in regard to display control and the way content should be published.

A very broad range of techniques have been studied to enable people to submit content for public displays. One of the earlier examples, the Plasma Poster [9], allowed people to submit photos, text, and web pages using email or a web form. SMS and MMS have also been extensively used as an interaction technique for the spontaneous generation of content. For example, the Joe Blogg project [10] includes a display designed in the form of an interactive artwork where people can send pictures and text messages through MMS or SMS. Hermes [11] explored the use of Bluetooth to enable users to send pictures and other media to a display. The use of Bluetooth names as an interactive feature has been described in [12] as an essentially opportunistic alternative that is easily available on a broad range of mobile device. It allows people to enter predefined commands in the Bluetooth name of their mobile phone. When that person approaches a display, these commands can be obtained and interpreted as part of the person's preferences. These and many other interaction studies (please refer to [13] for a comprehensive analysis of interaction alternatives) have mainly addressed the challenge of how to enable a person to approach a public display and spontaneously generate and submit content that somehow ends up being shown on the display. Our goal is to move beyond the interaction itself and focus on shared publication paradigms that enable people to understand and control what happens after content reaches the display system.

\section{INSTANT PLACES}

As part of our on-going work on situated displays, we have developed Instant Places as a web-centric platform for place-based screen media that explores new paradigms for situated publication in public displays. The main system entities are the places where the displays are located, the personal identities that allow people to control what they publish on the displays, and the applications that show content on the displays.

A place may have one or more displays and represents a symbolic environment that provides a meaningful context for situated social interaction, e.g. a restaurant, a shopping center, an office, or a city park. A place owner decides which content to accept on the respective displays, but we assume that place owners accept to integrate user-generated content into their displays as a way to engage with place visitors.

Identities allow people to explicitly and systematically manage publication and self-exposure in public displays. Through the Instant Places web site, people can create their identity and then use it to publish content either by attaching pins badges to their identity or by creating posters for distribution across the display network. The presence of an identity in a place is signaled through a check-in procedure available in the Instant Places mobile client, which is currently an Android application.

Multiple display applications can be created and selected for being used on the displays, where they should be able to adapt their behavior according to the available resources and current circumstances of that place. The system handles sensing and interaction information associated with places, e.g. current check-ins and user data, and provides an integrated API from which applications can obtain this information. Applications will also have access to data shared in that place by users, e.g. pins and posters, so that they can create visualizations of that data.

\section{A. Pin badges and posters}

The idea behind a publication paradigm is to frame, under a single and well-understood concept, a particular way to establish the relationship between content, identities, places and applications. Instant Places supports two types of publication paradigms: pin badges and posters. Their initial design was inspired by the respective non-digital counterparts, which are extensively used to express identity in general, announce events, support causes, advertising or identification with social groupings or organizations. The use of existing paradigms as a design inspiration is particularly interesting, in part because they offer known metaphors for a diverse range of publication scenarios in a way that people can already recognize, and in part because the type of communication practices that they enable is already embedded with multiple forms of locality and social rules that may be leveraged for this new medium. The two paradigms considered complement 
each other in the type of usage that they support, as they provide very different models for thinking about publication in public displays.

A pin badge refers to a particular institution, cause, campaign, sports teams, artist or brand that people may identify with. On the Instant Places web site, people can associate pins with their identities by going to their account and selecting from a list of predefined pin badges. A pin badge is composed by visual icon, a name, a set of tags, and a set of content sources from which it should be possible to generate screen content, e.g. a YouTube channel, a Flickr photo collection or a blog feed. Pins are not exactly a mechanism for user-generated content, in the sense that the pin user and the entity behind the pin content are not normally expected to be the same. Instead, pins work more on a crowdsourcing model in which users express support or interest to particular content sources. As people check-in to places, this information may be exposed as part of their identity and used to increase the popularity of the content associated with those pins.

Many applications could be created to explore the information associated with pin badges. For this study, we have created the Football application represented in Fig. 1, which displays content associated with the teams supported by place visitors through the use of pin badges. To allow people to express their football preferences, we created a pin collection with the complete set of football teams in the Portuguese premier league. When a person checks-in at a favorite place, if he or she has selected a pin corresponding to a football team, the content associated with that team is shown on the display. The application also shows the football pins being used by people at the place, thus providing a global view of the preferences of the place visitors. When there are no pins being exhibited by checked-in people, the application iterates over the most popular pins.

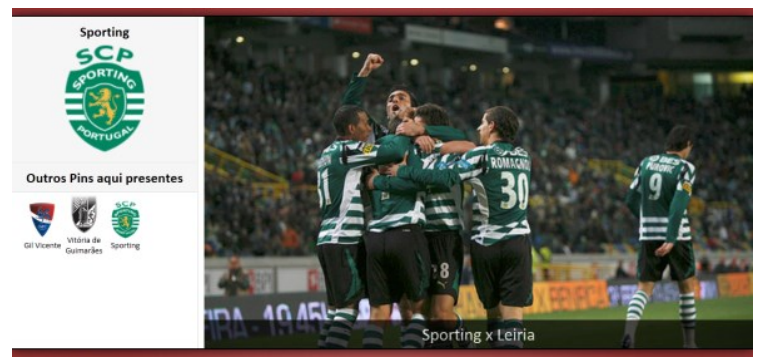

Fig. 1. Football application being driven by visitor's pins

A second pin collection was also created to represent the Top 20 Artists from the Rolling Stone Magazine. These could be selected by users, but since there was no application to exhibit them, they were never displayed. The sources associated with pins are assumed to be trusted publishers, and, therefore, moderation is not so much about preventing offensive content but more about aligning the content that people may call through their use of the pin badges with what may or may not be seen as appropriate for a particular place.
A poster refers to a media item with content for public displays that any user can create. The authoring process is done at the Instant Places web site and is very simple: upload the poster picture, give it a short title and description, and schedule its availability for display (currently limited to one month from the poster creation date). The poster application, represented in Figure 2, was used to iterate over the set of posters accepted at a place, showing the poster content along with the title and the identity name of the author.

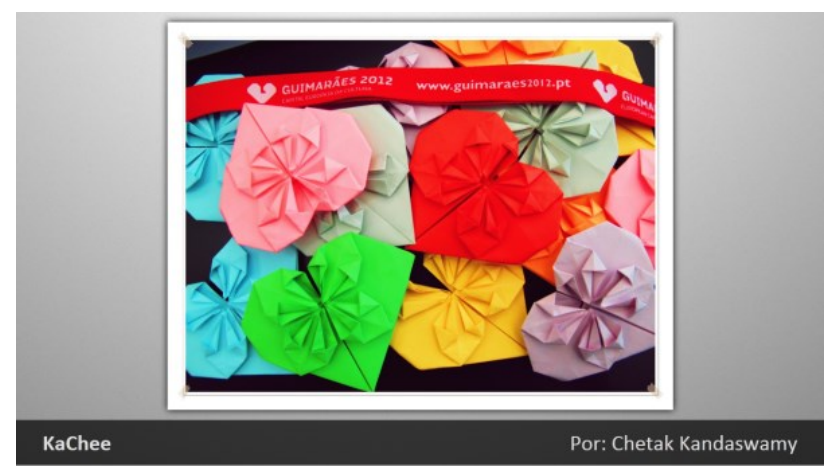

Figure 2. Posters application exhibiting an artwork

Place owners can select which posters to approve by going to their place web page on the Instant Places web site and selecting posters from a list of available posters. Posters can be recommended at a particular place through the Instant Places mobile app, in which case they will be shown at the top of that list. When a place owner approves a poster, that poster becomes immediately available for presentation by the Posters application in the local displays. Once a poster is published by a person, it can be removed by the author, but it cannot be changed. This aims to facilitate the moderation process by providing a permanent reference to the content that is shared across the entire system.

\section{DEPLOYMENT SETTING AND DATA COLLECTION}

\section{A. Living Lab on Situated Displays}

The Living Lab on Situated Displays is a set of urban display installations in the Minho region in Portugal, in which we grounded our study. This Living Lab is an ongoing initiative that started in 2008 with the exploration of Bluetooth-based interactions with public displays [12]. Since then, we have made multiple deployments of public displays in urban context at various types of locations and with diverse research goals.

The results reported in this article refer to a specific public trial occurring in 2012 over a period of 6 months and involving 10 public displays. The Instant Places system was progressively deployed across these sites during the first 4 months of the trial, and fully deployed at all location for the last 2 months of the trial. The deployment included 4 displays at our University ( 1 in a bar, 1 in a library and 2 in our own department), 3 deployments in public cafés in the city center of Guimarães, 2 deployments in schools and another one in a public library (c.f. Fig. 3). These diverse locations 
provided very different publics to the system, not only because of their different characteristics, but also because some of these spaces served very different crowds at different times. They are also associated with very diverse organizational realities.

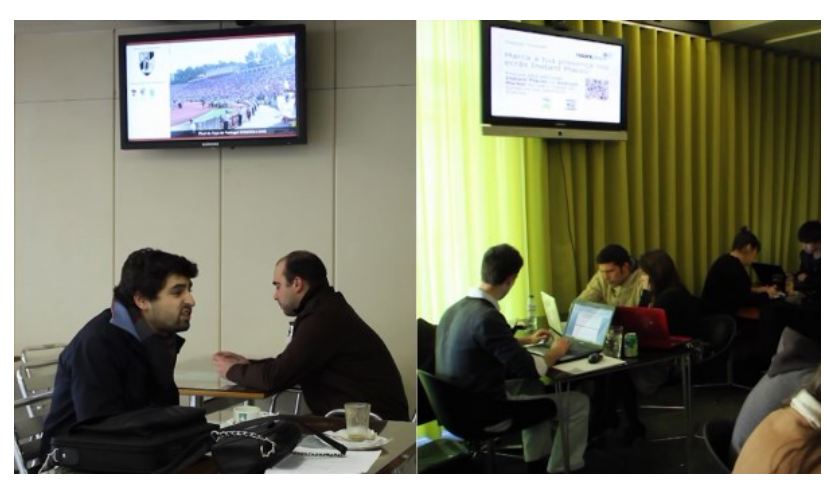

Fig. 3. University Bar (left) and Café-Concert (right)

Participants were invited to use the system through usage instructions that were occasionally presented on the display and also in small postcards that were distributed at the deployment spots.

\section{B. Data collection}

As part of this trial, we used a broad set of data collection techniques, combining qualitative and quantitative approaches.

At month 4, we invited the 59 users that were registered at that time to answer an online survey. The survey included questions regarding the motivations for registration, the users' first impressions with the system, and the main obstacles they had found in trying to use Instant Places. We collected 9 responses, 7 males and 2 females, aged between 23 and 37.

At month 6, we conducted semi-structured interviews with 5 place owners, 6 users of the system and 6 regular viewers of the system who had not become users. The interview scripts were slightly different for each of these groups, but the themes were essentially the same and were concerned with the practices around each of the two publication paradigms and the perceptions about authorship, moderation and appropriateness. All participants were shown an informed consent form that explained the purpose of the study. Interviews were recorded and later transcribed. To help contextualizing the interviews, these were conducted at the locations where participants had had contact with the system.

We also gathered quantitative data from usage logs that have been automatically collected since the beginning of the trial. The logs included information about all the main interaction events (e.g., poster publications, check-ins, pin selection, etc.). Information about mobile application installs was obtained from Google Play.

\section{V.RESULTS}

During the 6 months of the study, a total of 106 user accounts were created, all of them based on either
Facebook or Google profiles. The Instant Places Android App registered 63 installations, 20 of which were still active at the end of study period. 21 unique users generated 193 check-ins across 7 different locations. A total of 303 posters have been created during the trial, of which 127 were created by our research team to stimulate engagement and create a content baseline. The other 176 posters were created by 55 unique users, 4 of which place owners. 27 unique users added 63 pins to their profile.

We will now look in more detail into the results of the trial and analyze their meaning across three main themes: practices, which explores emergent behaviors around the two publication paradigms; situatedness, which explores the ways in which the publication paradigms enabled the association between published content and specific situations, i.e. locations, time or circumstances; and moderation and trust, which explores the role that the social layer can have in establishing reputation and appropriateness in the publication process.

\section{A. Publication practices}

A key goal for this trial was to uncover emerging practices around the effective use of the publication paradigms to inform the evolution of the systems and the design of additional paradigms and tools. All usage reported in this study occurred spontaneously as the result of the system availability across the 10 displays. We did not engage in any active form of recruitment, nor did we give prescriptive instructions for specific usages of the system. The 63 pin selections and the 176 posters that were created may thus be seen as genuine attempts to appropriate the possibilities offered by Instant Places.

The 63 pins selections were done by 27 distinct users (an average of 2.33 pins per user). Most of the selected pins (39) were picked from the football collection, which is not surprising since those were the only ones gaining visibility through the football application. The selected football pins were mainly from the two local clubs (Guimarães and Braga, with 14 picks) and from three major national teams, with 23 picks), reflecting the general behavior of the Portuguese football fans population of that geographical region. The selection patterns around Music pins were very different: Almost all pins were picked at least once, but none of them exhibiting a clear preference.

Most users seem to have understood the role of pins as an expression of personal preferences: "When we saw the pins, we knew it was about football and that we should select the team of our preference" [U1]. However, pins did not manage to catch the same degree of user engagement that we observed with posters, and two main reasons have emerged in the interviews. The first is that the pin collection was very small and football centric. While football is clearly a hot topic in this cultural setting, there were still many people who felt they did not identify with the available pins "I have only used one once because there were only pins I do not identify with very much" [U2]. Other users expressed a desired to have or even create pins that were almost personal, like a user who had his own band or a user who wanted a pin of his 
neighborhood. The second problem is that many people failed to establish a clear association between their pin choices and the content shown by the football application. "I thought the pins would have some impact on the system, perhaps the most popular ones would be shown on the display. At the time, I have chosen one, but I could not really understand its purpose beyond the characterization of the user profile." [U4]. This was made even worst by some of our design decisions that made it very difficult for pins to actually influence the display behavior. More specifically people had to have selected football pins, they needed to check-in through the Android app, and they had to indicate the place as favorite to allow those pins to be shared with the place. All these created an excessive large distance between selecting a pin and actually having it on the display.

The analysis of the practices around poster creation has benefited from the fact that they have generated considerably more user engagement. We have analyzed the types of content in the 176 posters created by users in the deployment and aggregated them into the informal categories represented in Table I.

The most popular category involved some form of support to football teams and, occasionally, other sports (32 posters). The European Football Championship that took place during the trial may have strongly influenced these numbers, but the key observation is that this is a category that does not correspond to any immediate and obvious reward for the person publishing the poster, other

TABLE I.

POSTERS PUBLISHED BY CATEGORY

\begin{tabular}{|l|c|c|}
\hline \multicolumn{1}{|c|}{ Poster category } & Posters & \% \\
\hline Fan: football/other sports & 32 & 18.2 \\
\hline Advertising (events) & 31 & 17.6 \\
\hline Advertising (institutional) & 26 & 14.8 \\
\hline Advertising (personal) & 17 & 9.6 \\
\hline Cooking suggestions & 15 & 8.5 \\
\hline Fan: music/actors & 14 & 8.0 \\
\hline Personal artistic expressions & 14 & 8.0 \\
\hline Other (errors and tests) & 8 & 4.5 \\
\hline Fan (cars, games, others) & 6 & 3.4 \\
\hline Humor & 6 & 3.4 \\
\hline Personal photos & 3 & 1.7 \\
\hline Causes & 3 & 1.7 \\
\hline Advertising (brands) & 1 & 0.6 \\
\hline TOTAL & 176 & 100.0 \\
\hline
\end{tabular}

than some sense of identification with a community.

In the case of more niche hobbies, e.g. cars, games (6) or music bands and actors (14), self-expression and the desire to make a personal statement were probably the main motivations. "I have made a poster about a concert that I have really enjoyed and that I wanted to share with other people." [U4]. "It is great to announce certain things to which I am associated with. With Facebook, only my friends get to see it, with Instant Places multiple people can see it" [U2]. Similarly, the cooking category (15), which was popular at one of the schools, was mainly a form of personal expressions about favorite foods than useful instructions.

Advertising events (31) included mainly parties, school events, sport events and music concerts. Some of these events posters were created by place owners who saw the system as a tool for communicating with their community. "I announce live music events and also other types of events such as the launch of a book we had the other day or the availability of a new product in our menu" [O2]. In addition to events, institutions have also used the medium to advertise courses and create awareness about local activities (26). "It is interesting for us to advertise our courses, but also to advertise the many great things happening in our school, such as the world championship in robotics and other prizes that we won" [O5]. Similarly, individuals have also appropriated the displays for personal advertising (17). Two photographers have created posters based on their portfolio, a book author advertised his recently launched book and some students have announced their project exhibitions. We also had a poster corresponding to the type of commercial advertising that is often associated with brands.

The last major category involved the display of artistic works (14) by non-professionals. In most of these cases, the publisher was not the artist himself or herself, but someone using the displays to show something they appreciated, e.g. from a daughter or a friend (c.f. Fig. 3). At one of the schools, students appropriated posters to show some of their school works: "it is an extra motivation when someone has made a nice work to have it shown to the community. The colleagues will see it, they know the person and these will normally result in conversations and comments about the work."[O1]

We also asked participants about their content creation practices. The format used for the posters (an image and a text title) was clearly limiting, but we did not expect this to be a problem, since most users were having their first experience with content creation for public displays. Even though this was indeed the case for most participants, at one of the schools, the involvement of a local community of participants led to early attempts for differentiation These students have quickly found themselves trying to make their poster more attractive to differentiate it from those of the other colleagues, leading to a series of evolving practices and increasingly more sophisticated designs. At first, this meant realizing the importance of large fonts for visibility on the public displays, but then it quickly evolved to trying to add color effects to make the posters more attention grabbing. "To make the poster more attractive, we add image effects, we add a contour, or we change the color of the photo. It grabs peoples' attention" [U1]. A photographer wanting to expose some images from his portfolio, and being confronted with our one image limitation per poster, 
created interesting photo collages, each made of a themed composition of several related photos and taking full advantage of the horizontal display.

The use of a widely known format, such as images, has revealed two additional insights regarding content creation. The first was the importance of allowing everyone to leverage upon their favorite tools to create content. Participants reported using PowerPoint, Paint, Word and even combinations of these, and this flexibility in tool selection may have been crucial to enable many of them to become publishers. The other insight was about the importance of content repurposing, as many of the images used for the posters were clearly downloaded from online sources and published as is. This was very often the case with events, many of which would already have some form of poster. In a world-wide campaign against soldier children occurring during the study, the fact that the campaign itself already provided poster images in various sizes and languages may have been fundamental in leading one of our users to repurpose that content to create a poster in Instant Places. This facilitation is a trend that we envision may considerably contribute to increase media sharing on public displays. However, despite being very convenient, it also had some limitations. In some cases, the information on the repurposed content was too small to be perceptible from the normal viewing distance of the display, and most of the pictures used had a vertical orientation, thus failing to take full advantage of the horizontal screen orientation that was common to all our displays.

Overall, the main problem identified by participants in relation to the publication paradigms has been the lack of feedback on publication: "we do not perceive the advantage of creating these posters. [In Facebook], we have likes, we have some feedback" [U3]. Not having a sense of when or even if their content had ever been presented was clearly frustrating for participants. Some participants have also expressed the desire to make their content shown on specific situations. This was very clear with the students of one of the schools that only really cared about having their poster shown during the breaks when all their colleagues were there and could see it. In these situations, any other content would just be perceived as taking time away from what really mattered.

\section{B. Situatedness}

A key element of situated publication is how to enable the association between user-generated content and particular situations, i.e., how to define the spatial, temporal or circumstantial scope of publication. Since digital content can potentially be published anywhere and as many times as wanted, the research goal is to understand what type of properties may be more effective in incorporating situatedness as an integral part of any publication act in a way that helps to correlate the relevance of that content with particular situations.

In regard to the spatial scope of poster publication, we have clearly observed a tension between content that was perceived as strongly locative and content that the authors simply wanted to disseminate to as many locations as possible, as suggested by a place owner: "The important is to spread the message to multiple locations because if only presented here it is very limiting" [O5]. This tension became particularly evident when we changed the poster distribution policy in month 4 of the trial. Until then, posters were created on the web site, but then, their creator would have to check-in at the places where it should be distributed. This approach was a direct metaphor for what happens today with physical posters or leaflets and matched very nicely with the type of locativeness that we were looking for. However, we quickly realized that it was also too restrictive and cumbersome. In early user interviews, some users reported engaging in successive check-ins at multiple places, even if not physically there, just to be able to quickly disseminate posters to all those locations. Additionally, many people were never even able to recommend their posters because they did not have an Android device. Based on these initial observations, we changed the poster distribution process. After month 4, posters would immediately become available anywhere as soon as they were created on the website, and place owners could select whichever they wanted from a list of available posters. Users could still check-in at a place and recommend a poster, in which case the relevance of that poster would be increased when being shown to the local place owner for possible selection.

When we made this change, it became clear that many users had never even considered the existence of other similar displays at other locations and some of them were not happy about the new distribution model. This was particularly evident at one of the schools in the trial, where teachers were stimulating students to post content, but then become worried that such content could be shown in other places: "We should be able to control that what is published here stays here. We have posters with student photos in school activities, and having that shown in places we do not even imagine raises many questions in terms of usage and security" [O1]. A similar concern was also expressed by some individual users, who were not comfortable with the idea that the posters they had created to be shown at a specific location could now be public and shown anywhere.

However, views on this issue seemed to be strongly dependent on the nature of content, and even the same school considered that in some cases dissemination outside the school would be the right approach: "It would be great if you could set that posters were only for the school because then we could control what was public or not, although in some cases it would be interesting to have some content disseminated to other schools or even to the local community as when we want to announce open events." [O1].

Time is another key dimension for situatedness. While posters included a validity range that defined for how long they would still be presented, most users have simply used the maximum allowed time, a month, apparently without giving much thought to the issue. The 
perception about content validity was only strong in regard to posters announcing events with a scheduled date: "If it was an event, I would try to set validity to the day of the event, otherwise I always used the maximum time. It gives us a month, isn't it?'. A more sophisticated management of situatedness occurred with a poster supporting the national football team on their participation in the European Championship. Even though the end date of the championship was known, the date when the team would be eliminated from the competition was not, and therefore posters were carefully set until the next game and, then, in case of victory, were submitted again to avoid the potential embarrassment of placing a support message after the team had been eliminated.

In regard to pin badges, situatedness was essentially a reflection of the people checked-in at any moment and the pins they exhibited. While the system allowed people to choose whether or not to show their pins on a particular place through the selection of different personas, we have not observed any cases where people seemed to be trying to control their self-exposure based on their own interpretation of the situation around a particular display.

\section{Moderation and trust}

The various forms of social negotiation that are necessarily involved in the shared use of public displays, should also be a key element in shaping publication paradigms, particularly in regard to moderation and trust.

Most place owners expressed that moderation would be fundamental for their use of the system, but effective moderation practices differed substantially between place typologies. For example, at the schools, moderation was perceived as fundamental, not only by teachers but also by students. Other places, such as bars, assumed a much more relaxed attitude towards poster moderation and some of them were even comfortable with the idea of not having moderation at all, as long as they could remove any offending posters. When asked about the best way to deal with explicit requests from users to present a poster that they considered inappropriate, all place owners supported the idea that they should simply be ignored, instead of generating any type of explicit rejection message: "I do not think that people should be notified. It could generate a sense of unease and if the person sees that her poster is not being shown and really wants to know why, she can ask the person in charge". [O3]

The existence of moderation seems to have been enough to formulate a shared sense of what was acceptable and we have only registered a refused poster, when clients of a bar wanted to publish a poster poking at other clients and the owner told them it would not be appropriate. Still, there was often a tension between what was valued by users of the display and what the display owner perceived as valuable to the place. The interviews at school [O1] were particularly suggestive. Students seemed to appreciate the more informal nature of the display and the fact that it allowed them to channel their desire to express their tastes for sports, music or motorcycles. The teacher playing the role of display owner, while seeing such freedom as a positive to engage students with the system, expressed a desire to have posters with more quality, addressing less mundane topics, and that could instead be seen as more relevant to the whole school community.

In regard to pins, moderation was much narrower, as content was assumed to be trusted. Still, we have observed concerns with the set of pins that could be displayed. This was the case with football pins from rival teams from nearby cities. While not offensive per se, the display of those pins in particular places was clearly indicated as being provocative. This means that even when considering a particular application selected by a place owner to show themed content on his or her display, it may still be relevant to have the possibility to filter certain pin badges that may not be appropriate for that particular place.

Identity has also emerged as an important part of the social negotiation process. The use of personas is supported by Instant Places to allow people to control the way their presence is exposed, and when designing the poster application, we considered the possibility of using those nicknames to indicate the author of a poster being presented. In the end, we decided to use the normal identity name based in part of the assumption that publishing a poster should be seen as a public act and that having the identity name on the display would constitute an important form of accountability that could prevent many sorts of inappropriate behavior. The results of the trial suggest that this was the right option. From the perspective of place owners, it was often mentioned in the interviews that when selecting posters for presentation at their sites, the name of the author and the recognition that it was someone familiar was often a key selection criteria. "Recognizing the person as one of our students was very important. Even if the poster was not directly linked with the school, it was still content from someone from our community, and the students would be very satisfied to see their poster presented to others" [O1].

Interestingly, many poster creators were very positive about having their public name displayed. We cannot know if anyone may have decided not to create posters because of the lack of anonymity, but some participants perceived the public display of their name as adding credibility to the poster and being rewarding to them: "I liked seeing my name when I made my first poster. It was there and yes I liked it seeing my name". [U4]. Still others can see some advantages in anonymity, at least in regard to what is shown on the display. "I like to have my posters associated with my name when I want to show that I am the author. However, there should be an option to not show the name, as the poster needs to be approved anyway". [U2].

While the relatively low number of displays was not sufficient to create a strong sense of network, some place owners become mutually aware and engaged in content exchange practices that aimed to increase the reach of the 
posters. "I received a phone call from [O2] asking if I could show their posters on my display and they would do the same with mine" [O4]. This type of back-channel interactions may hint at the type of social connections that may be embedded into new publication paradigms.

\section{CONCLUSIONS AND FUTURE WORK}

This article described the results of the deployment of an urban network of displays that aimed to explore two new publication paradigms for user-generated content in public displays: pin badges and posters. Overall, the two publication paradigms were both appreciated by participants, but posters have clearly generated much more appropriation that pin badges. The lack of feedback and the ability to pull content in specific situations have emerged as the most missed features in our implementation of these publication paradigms. They have clearly been indicated by many users as a frustrating part of the publication experience and have thus become a major target for future work. The fact that only an Android client was used was also reported as a limitation to the use of the system by several potential participants.

The results are also representative of the variety of perspectives that people can have about any of the topics that have been explored. Clearly, publication paradigms for public displays need to be designed with enough flexibility to accommodate diverse social contexts and reflect the sophisticated nature of how Humans connect with places. In particular, these two publication paradigms should be seen as starting points for the evolution towards more, and more sophisticated, publication paradigms. The marginal practices that have been identified may constitute initial hints for that evolution. For example, the especially complex role of time in event-related posters seems to suggest a specific type of poster tailored to those specificities. The different views on the locativeness of posters hints at a specific concept of poster that is inherently locative and cannot be taken away or shown at other locations.

The generalization of these findings to large scale display networks is also challenging, mainly because many emergent behaviors may only occur after a certain critical mass of users, displays or applications is reached or after practices evolve over time. The scalability of the paradigms that have been studied may also be questioned when we consider very large display networks, but the explicit representation of social connections between places and also between people and places may provide an interesting path for dealing with those challenges. References to "people from our community" or "places with which we could share content" suggest that a formal representation of these connections in the publication paradigms could have a strong impact on publication practices, enabling new forms of situatedness and providing the ground for crowdsourcing models that may improve the scalability of these publication processes.

\section{ACKNOWLEDGMENTS}

The research leading to these results has received funding from the European Union Seventh Framework Programme (FP7/2007-2013) under grant agreement no. 244011.

\section{REFERENCES}

[1] N. Davies, M. Langheinrich, R. Jose, A. Schmidt, and R. José, "Open Display Networks: A Communications Medium for the 21st Century," Computer, vol. 45, no. 5, pp. 58-64, Mar. 2012

[2] J. Müller, D. Wilmsmann, J. Exeler, M. Buzeck, A. Schmidt T. Jay, A. Krüger, H. Tokuda, M. Beigl, A. Friday, A. Brush, and Y. Tobe, "Display Blindness: The Effect of Expectations on Attention towards Digital Signage," in Pervasive Computing, 2009, vol. 5538, pp. 1-8.

[3] E. M. Huang and E. D. Mynatt, "Shared Displays for Small Communities: Optimizing for Privacy and Relevance," in Workshop Public, Community and Situated Displays, 2002.

[4] J. Müller, F. Alt, D. Michelis, and A. Schmidt, "Requirements and design space for interactive public displays," in Proceedings of the international conference on Multimedia, 2010, no. Figure 1, p. 1285.

[5] N. Memarovic, M. Langheinrich, E. Rubegni, A. David and I. Elhart 'Designing 'Interacting Places' for a Student Community using a Communicative Ecology Approach," in 11th International Conference on Mobile and Ubiquitous Multimedia, 2012.

[6] R. Schroeter, M. Foth, and C. Satchell, "People, content, location," in Proceedings of the Designing Interactive Systems Conference on - DIS '12, 2012, p. 146.

[7] F. Alt, N. Memarovic, I. Elhart, D. Bial, and A. Schmidt, "Designing shared public display networks: implications from today's paper-based notice areas," Pervasive, pp. 258-275, 2011.

[8] A. Friday, N. Davies, and C. Efstratiou, "Reflections on Long-Term Experiments with Public Displays," Computer, vol. 45, no. 5, pp. 34-41, May 2012.

[9] E. Churchill, L. Nelson, L. Denoue, P. Murphy, and J. Helfman, "The Plasma Poster Network," Public and Situated Displays Social and Interactional Aspects of Shared Display Technologies, pp. 233-260, 2003.

[10] K. Martin, A. Penn, and L. Gavin, "Engaging with a situated display via picture messaging," in CHI 06 extended abstracts on Human factors in computing systems CHI 06, 2006, pp. 1079-1084.

[11] K. Cheverst, A. Dix, D. Fitton, C. Kray, M. Rouncefield, C. Sas, G. Saslis-Lagoudakis, and J. G. Sheridan, "Exploring bluetooth based mobile phone interaction with the hermes photo display," in 7th international conference on Human computer interaction with mobile devices services MobileHCI 05, 2005, vol. 111, pp. 47-54.

[12] R. Jose, N. Otero, S. Izadi, and R. Harper, "Instant Places: Using Bluetooth for Situated Interaction in Public Displays," Pervasive Computing, IEEE, vol. 7, no. 4, pp. $52-57$, Dec. 2008.

[13] R. Ballagas, J. Borchers, M. Rohs, and J. G. Sheridan, "The Smart Phone: A Ubiquitous Input Device," IEEE Pervasive Computing, vol. 5, no. 1, pp. 70-77, Jan. 2006.

\section{AUTHORS}

\section{Rui José.}

Rui José is an Assistant Professor at the University of Minho, in Portugal, where he leads the Ubicomp research group. Rui José received his $\mathrm{PhD}$ in Computer Science 
from Lancaster University in 2001. His research interests are in ubiquitous systems in general and particularly on situated displays. Rui José is a co-PI in PD-Net, a project funded under the FET-Open program to create the foundations for open displays networks as a new global communication medium for information access and interaction. Rui José is a member of ACM and its SIGCHI.

\section{Helder Pinto}

Hélder Pinto has been involved in several ubiquitous and pervasive computing research projects at the University of Minho. His interests range from Ubiquitous Computing to Human-Computer Interaction in general. The topic of his $\mathrm{PhD}$ (2008) was activity-based ubiquitous computing environments. In 2007, he joined Ubisign, a digital signage software company, where he has since been leading the R\&D department.

\section{Bruno Silva}

Bruno Silva is a Software Engineer and he is finishing his Master's degree in combinatorial optimization. He has been a member of the Ubicomp research group at University of Minho since 2008. He is a researcher in the PD-Net project, where he has worked on the design and development of the instant places platform.

\footnotetext{
Ana Melro

Ana Melro is a social sciences researcher. She has a degree and a master degree in Sociology, both form University of Minho. Ana Melro is currently a $\mathrm{PhD}$ student in Information and Communication in Digital Platforms at University of Aveiro. Her research interests include digital literacy and inclusion, screen uses, sociohistorical perspectives of new media appropriation and, more recently, ubiquitous systems in general. She is also a member of the research group CETAC.MEDIA.
} 